\title{
Container evolution and dynamics of cluster formation
}

\author{
Yasuro Funaki, ${ }^{*}$ \\ ${ }^{1}$ College of Science and Engineering, Kanto Gakuin University, Yokohama 236-8501, Japan
}

\begin{abstract}
We introduce the so-called Tohsaki-Horiuchi-Schuck-Röpke (THSR) wave function to describe various nuclear cluster states. Its importance, applicability, and usefulness are extensively discussed in this report. It is demonstrated that the THSR wave function provides a "container" picture for cluster structures and even an evolution of the container, for a couple of typical examples, such as ${ }^{20} \mathrm{Ne},{ }^{12} \mathrm{C}$, and ${ }^{16} \mathrm{O}$ nuclei.
\end{abstract}

\section{Introduction}

The cluster formation plays an important role in light nuclei. The $3 \alpha$ cluster structure in ${ }^{12} \mathrm{C}, \alpha+{ }^{12} \mathrm{C}$ cluster structure in ${ }^{16} \mathrm{O}$ and $\alpha+{ }^{16} \mathrm{O}$ cluster structure in ${ }^{20} \mathrm{Ne}$ are the typical examples and extensively studied by using various cluster models [1]. In the past two decades, alphaparticle condensation has been extensively studied theoretically and experimentally. Although providing direct observatory evidence is still an open question [2-4], many theoretical calculations predict the existence of the $3 \alpha$ and $4 \alpha$ condensate states in ${ }^{12} \mathrm{C}$ and ${ }^{16} \mathrm{O}$, respectively, in which all $\alpha$ clusters weakly interact with each other with a dilute gaslike configuration, and occupy an identical orbit of a meanfield-like potential [5-12].

On the other hand, the ordinary non-gaslike cluster states like the $\alpha+{ }^{16} \mathrm{O}, \alpha+{ }^{12} \mathrm{C}$ inversion doublets, linearchain $\alpha$-cluster states, etc. are completely different from the gaslike cluster states. They had been understood by a concept of localized clustering, in which all clusters are in a geometric arrangement. However, more recent works have required us to modify the basic idea of understanding the ordinary cluster states. In Ref. [13], we introduced a microscopic $\alpha+{ }^{16} \mathrm{O}$ cluster model wave function, which demonstrates a nonlocalized motion of the $\alpha$ and ${ }^{16} \mathrm{O}$ clusters. They proved that the model wave function coincides with the full solution of $\alpha+{ }^{16} \mathrm{O}$ Resonating Group Method (RGM) equation of motion for all the $\alpha+{ }^{16} \mathrm{O}$ inversion doublet band states. Similar results are also obtained for $3 \alpha$ and $4 \alpha$ linear-chain states [14]. All these results lead to the idea that dynamically mutual clusters are confined in a "container", whose shape and size are flexibly conformed, in a nonlocalized way. This new concept of the so-called "container" picture modifies the preceding understanding of nuclear clustering, since the localized clustering has been an important basis to understand the ordinary (non-gaslike) nuclear cluster structures. The spatial localization of clusters seems to appear when the size of container is very small, due to the effect of Pauli principle acting on clusters in between, as a kinematical effect.

*e-mail: yasuro@kanto-gakuin.ac.jp
In this contribution, the study of ${ }^{20} \mathrm{Ne}$, which urges us to introduce the container picture, is briefly reported, in which the so-called Tohsaki-Horiuchi-Schuck-Röpke (THSR) ansatz is extended. The extended THSR ansatz is applied to the excited states above the Hoyle state in ${ }^{12} \mathrm{C}$, in the following section [15-17]. Finally the same ansatz is applied to ${ }^{16} \mathrm{O}$ and the cluster evolution in ${ }^{16} \mathrm{O}$, from the ground state, $\alpha+{ }^{12} \mathrm{C}$ clustering, to the $4 \alpha$ clustering, is investigated with a new concept, "container evolution" [18].

\section{2 "Container" picture in ${ }^{20} \mathrm{Ne}$}

${ }^{20} \mathrm{Ne}$ nucleus is one of the most typical clustered nuclei, where the observed ground-state band and $K^{\pi}=0_{1}^{-}$band are considered to form the inversion doublet band arising from the hetero-polar di-nucleus configuration [19] of $\alpha+{ }^{16} \mathrm{O}$ cluster structure. The observed large $\alpha$ reduced width and degenerate nature of both bands cannot be explained without assuming a parity violating spatial localization of $\alpha$ and ${ }^{16} \mathrm{O}$ clusters. The inversion doublet band states of $\alpha+{ }^{16} \mathrm{O}$ cluster structure are therefore best described by the microscopic $\alpha+{ }^{16} \mathrm{O}$ cluster model, i.e. $\alpha+{ }^{16} \mathrm{O}$ RGM, or equivalently $\alpha+{ }^{16} \mathrm{O}$ Brink-Generator Coordinate Method (Brink-GCM). The latter is to solve the following Hill-Wheeler equation,

$$
\sum_{j}\left\langle\Phi_{\text {Brink }}^{J^{\pi}}\left(R_{i}\right)|\hat{H}-E| \Phi_{\text {Brink }}^{J^{\pi}}\left(R_{j}\right)\right\rangle f\left(R_{j}\right)=0,
$$

where, $\Phi_{\text {Brink }}^{J^{\pi}}(R)$ denotes the Brink wave function projected onto $J^{\pi}$ angular momentum and parity, with $R$ the relative distance parameter between the $\alpha$ and ${ }^{16} \mathrm{O}$ clusters. The Brink-GCM wave function is expressed as follows:

$$
\Phi_{\mathrm{GCM}}^{J^{\pi}}=\sum_{i} f\left(R_{i}\right) \Phi_{\mathrm{Brink}}^{J^{\pi}}\left(R_{i}\right)
$$

On the other hand, in Ref. [13], a hybrid-Brink-THSR wave function wave function was proposed as follows:

$$
\begin{aligned}
& \Phi_{\mathrm{THSR}}^{J^{\pi}}(\boldsymbol{\beta}, \boldsymbol{S}) \\
& \quad \propto \hat{P}^{J^{\pi}} \mathcal{A}\left\{\exp \left[-\sum_{k=x, y, z} \frac{8\left(r_{k}-S_{k}\right)^{2}}{5 B_{k}^{2}}\right] \phi(\alpha) \phi\left({ }^{16} \mathrm{O}\right)\right\},
\end{aligned}
$$


where $\hat{P}^{J^{\pi}}$ the projection operator onto the angular momentum and parity $J^{\pi}, B_{k}^{2}=b^{2}+2 \beta_{k}^{2},(k=x, y, z), \boldsymbol{r}$ the dynamical relative coordinate between the two clusters. All calculations are performed with restriction to axially symmetric deformation, that is, $\beta_{x}=\beta_{y} \neq \beta_{z}$ and $\boldsymbol{S} \equiv\left(0,0, S_{z}\right)$

Table 1. $E_{\min }^{J^{\pi}}$ is the energy to give minimum in parameter space, $\beta_{x}=\beta_{y}, \beta_{z}$, with $S_{z} \rightarrow 0$. The corresponding values of $\beta$ and $S_{z}$ in the hybrid model and the squared overlaps between the (normalized) hybrid wave functions $\Phi_{\mathrm{THSR}}^{J^{\pi}}$ and the (normalized) Brink GCM wave functions are also listed.

\begin{tabular}{cccc}
\hline$J^{\pi}$ & $\left(\beta_{x}=\beta_{y}, \beta_{z}\right)$ & $E_{\min }^{J^{\pi}}[\mathrm{MeV}]$ & $\left|\left\langle\Phi_{\mathrm{THSR}}^{J^{\pi}} \mid \Phi_{\mathrm{GCM}}^{J^{\pi}}\right\rangle\right|^{2}$ \\
\hline $0^{+}$ & $(0.9,2.5)$ & -159.85 & 0.9929 \\
$2^{+}$ & $(0.0,2.2)$ & -158.53 & 0.9879 \\
$4^{+}$ & $(0.0,1.8)$ & -155.50 & 0.9775 \\
$1^{-}$ & $(3.7,1.4)$ & -155.38 & 0.9998 \\
$3^{-}$ & $(3.7,0.0)$ & -153.07 & 0.9987 \\
\hline
\end{tabular}

In Refs. [13, 20], the authors found that the energy is most favoured when $S_{z} \rightarrow 0$ in two parameter space of $S_{z}$ and $\beta_{x}=\beta_{y}=\beta_{z}$, for all $J^{\pi}$ states. Since the hybrid BrinkTHSR wave function in Eq. (3) contains both limits of the Brink and THSR wave function, which are characterized by the parameters $S_{z}$ and $\boldsymbol{\beta}$, respectively. The fact that $S_{z} \rightarrow 0$ is energetically favored means that the pure THSR wave function is selected, i.e. strongly indicating that the "container" picture is favored, without giving any optimal relative distance between the two clusters. The variational calculations are furthermore put forward and they found that the pure THSR wave function gives almost $100 \%$ squared overlap with the Brink-GCM wave function for all the $J^{\pi}$ states of the inversion doublet band states. The results are summarized in Table 1 , where the single configuration of the pure THSR wave function giving the minimum energy in $\beta_{x}=\beta_{y}, \beta_{z}$ parameter space with the limit $S_{z} \rightarrow 0$, is taken. Actually, the table shows that the squared overlap values are $99.29 \%, 98.79 \%, 97.75 \%$, $99.98 \%$, and $99.87 \%$ for $J^{\pi}=0^{+}, 2^{+}, 4^{+}, 1^{-}$, and $3^{-}$states, respectively. These almost $100 \%$ squared overlap values of course mean that the single THSR wave functions are almost $100 \%$ equivalent to the corresponding RGM/GCM wave functions, and thus they can accurately describe the inversion doublet band states in ${ }^{20} \mathrm{Ne}$. We should then note that the THSR wave function never seems to provide any geometric configuration, like giving an optimal spatial placement of the two clusters, but gives rather a delocalized motion in a meanfield-like potential, which is referred to as a "container" picture.

Now many other studies show the correctness of this concept in other systems, which had been (before the advent of this new concept) considered to have localized clustering structures, such as the $3 \alpha$ - and $4 \alpha$-linear chain structure states, $\alpha+\alpha+\Lambda$ structure state in ${ }_{\Lambda}^{9} \mathrm{Be}$, etc. In our new interpretation, the linear chain states have a structure that the $\alpha$ clusters are confined in a prolately deformed container, and in ${ }_{\Lambda}^{9} \mathrm{Be}$, in which the density is made much higher than that of ${ }^{8} \mathrm{Be}$, due to the $\Lambda$ particle, the three clusters are confined in a small size container with deformation [21]. The concept has also been examined in many other systems, including neutron-rich nuclei, like ${ }^{9} \mathrm{Be}[22]$, ${ }^{10} \mathrm{Be},{ }^{12} \mathrm{Be}[23],{ }_{\Lambda}^{13} \mathrm{C}$ [24], ${ }^{9} \mathrm{~B}$ [25].

\section{Excited states above the Hoyle state in ${ }^{12} \mathrm{C}$}

The Hoyle state is the famous example of the $\alpha$ condensate state, in which the $3 \alpha$ clusters weakly interact with each other and are condensed into the lowest energy orbit. We here argue that there exist a number of other $\alpha$ cluster states above the Hoyle state, which we can qualify as the excited states of the Hoyle state. We investigate them by using the following extended THSR wave function (eTHSR),

$$
\begin{aligned}
& \Phi_{3 \alpha}^{J=0}\left(\boldsymbol{\beta}_{1}, \boldsymbol{\beta}_{2}\right) \\
& \quad=\hat{P}^{J=0} \mathcal{A}\left[\prod_{i=1}^{2} \exp \left\{-\mu_{i} \sum_{k=x, y, z} \frac{2\left(\xi_{i x}\right)^{2}}{b^{2}+2 \beta_{i k}^{2}}\right\} \prod_{i=1}^{3} \phi\left(\alpha_{i}\right)\right],
\end{aligned}
$$

in which the two different width parameters $\boldsymbol{\beta}_{1}$ and $\boldsymbol{\beta}_{2}$ are associated to the two Jacobi coordinates $\boldsymbol{\xi}_{1}$ and $\boldsymbol{\xi}_{2}$, corresponding to $\alpha$ - $\alpha$ and $2 \alpha-\alpha$ motions, respectively.

With this eTHSR wave function, one can get a much richer spectrum of ${ }^{12} \mathrm{C}$ by solving the Hill-Wheeler equation, with axial symmetry assumed and the four $\beta$ parameters taken as generator coordinates. In this calculations we effectively remove spurious continuum components by using a method of the so-called radius constraint method (see Refs. [16, 26-28] for details). In Fig. 1, the calculated energy spectrum is shown. One can see that besides the ground state band, there are many $J^{\pi}$ states obtained above the Hoyle state. All these states turn out to have large rms radii $(3.7 \sim 4.7 \mathrm{fm})$, and therefore can be considered as excitations of the Hoyle state. The Hoyle state can thus be considered as the "ground state" of a new class of excited states in ${ }^{12} \mathrm{C}$. In particular, the nature of the series of states $\left(0_{2}^{+}, 2_{2}^{+}, 4_{2}^{+}\right)$and the $0_{3}^{+}$and $0_{4}^{+}$states have recently been much discussed from the experimental side. The $2_{2}^{+}$state that theoretically has been predicted at a few $\mathrm{MeV}$ above the Hoyle state already in the early works of $3 \alpha$ Brink-GCM $[29,30]$ and $3 \alpha$ RGM [31] was recently confirmed by several experiments [32-37]. A strong candidate for a member of the Hoyle family of states with $J^{\pi}=4^{+}$was also reported by Freer et al. [38]. Itoh et al. recently pointed out that the broad $0^{+}$resonance at 10.3 $\mathrm{MeV}$ should be decomposed into two states: $0_{3}^{+}$and $0_{4}^{+}$ [39]. This finding is consistent with theoretical predictions where the $0_{3}^{+}$state is considered as a breathing excitation of the Hoyle state $[16,17,40,41]$ and the $0_{4}^{+}$state as the bent arm or linear chain configuration [17, 42, 43].

In Fig. 1, the $E 2$ transition strengths between $J$ and $J \pm 2$ states and monopole transitions between $0^{+}$states are also shown with corresponding arrows. We can note the very strong $E 2$ transitions inside the Hoyle band, $B\left(E 2 ; 4_{2}^{+} \rightarrow 2_{2}^{+}\right)=591 e^{2} \mathrm{fm}^{4}$ and $B\left(E 2 ; 2_{2}^{+} \rightarrow 0_{2}^{+}\right)=$ $295 e^{2} \mathrm{fm}^{4}$. The transition between the $2_{2}^{+}$and $0_{3}^{+}$states is also very large, $B\left(E 2 ; 2_{2}^{+} \rightarrow 0_{3}^{+}\right)=104 e^{2} \mathrm{fm}^{4}$. In Fig. 2, the calculated energy levels are plotted as a function of $J(J+1)$, together with the experimental data. There 


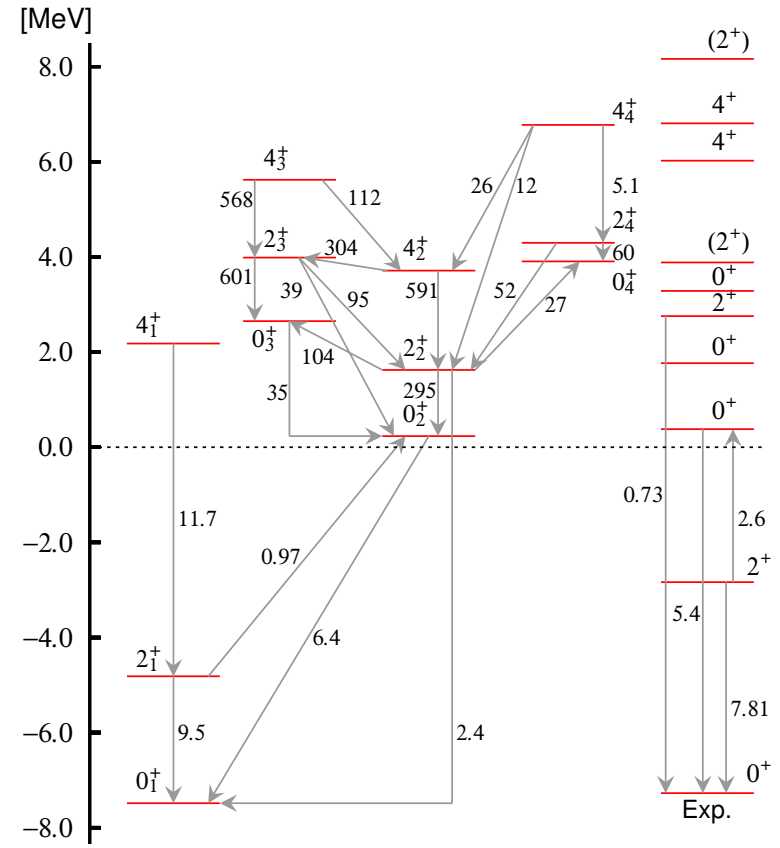

Figure 1. (Color online). Calculated energy levels and electric transition strengths are shown and compared with experiments.

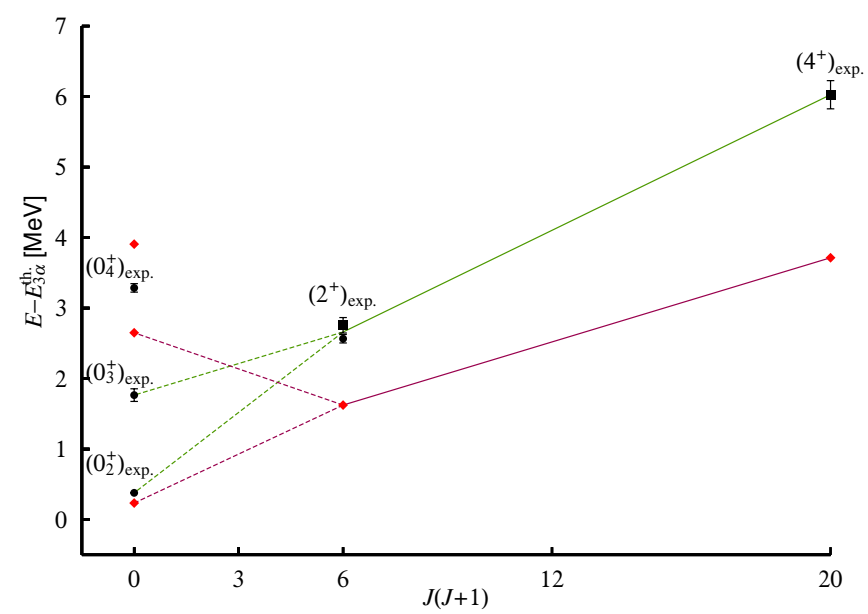

Figure 2. (Color online). The observed energy levels for the $0_{3}^{+}$, $0_{4}^{+}$, and $2_{2}^{+}$states in Ref. [32], and the $2_{2}^{+}$[37] and $4_{2}^{+}$[38] states are denoted by black circles and black squares, respectively. The calculated energy levels for the five states are denoted by red diamonds.

have been attempts to interpret this as a rotational band of a spinning triangle as this was successfully done for the ground state band $[44,45]$. However, the situation may not be as straightforward as it seems. This is because the two transitions $2_{2}^{+} \rightarrow 0_{2}^{+}$and $2_{2}^{+} \rightarrow 0_{3}^{+}$are of similar magnitude, and hence no clear band head can be identified. It was also pointed out in Refs. [42, 43] that the states $2_{2}^{+}, 4_{2}^{+}$ form a rotational band not with the $0_{2}^{+}$but with the $0_{3}^{+}$state. The line which connects the two other hypothetical members of the rotational band, in Fig. 2, has a slope, which
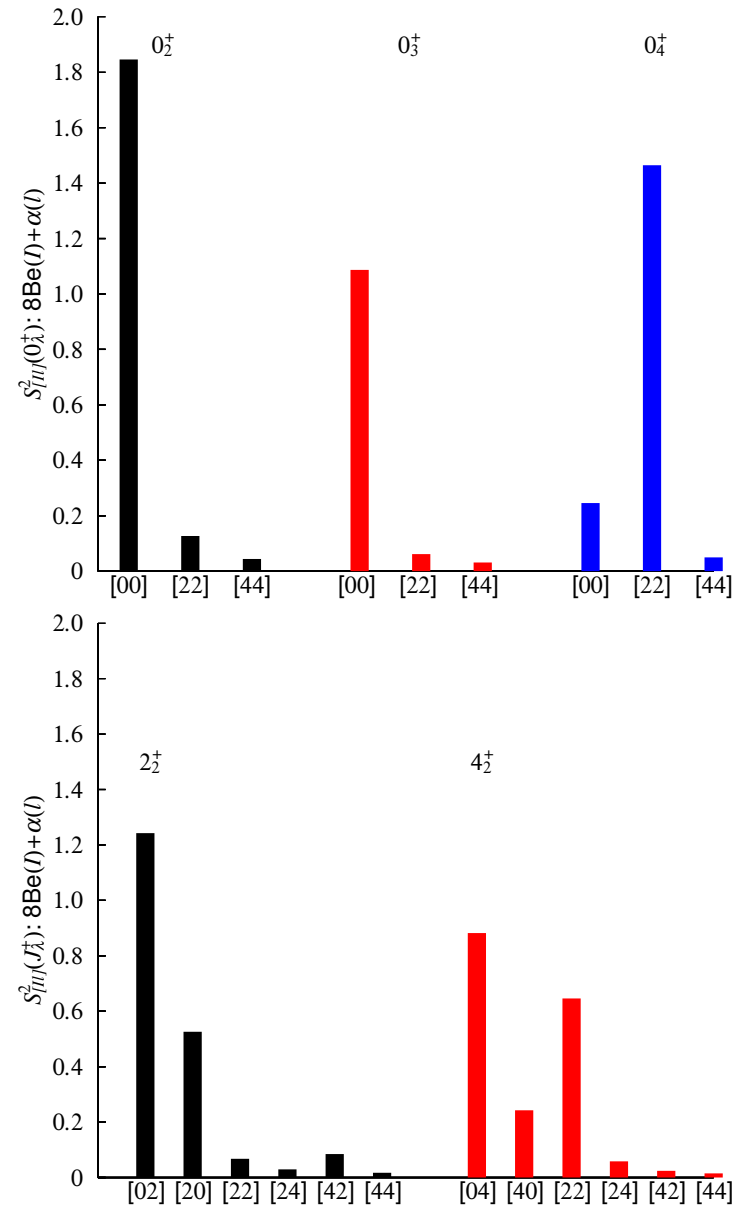

Figure 3. (Color online) $S^{2}$ factors of the excited states.

points to somewhere in between of the $0_{2}^{+}$and $0_{3}^{+}$states. The similar effect is also argued in the study of the $4 \alpha$ condensate and ${ }^{12} \mathrm{C}\left(0_{2}^{+}\right)+\alpha$ rotational band in ${ }^{16} \mathrm{O}[46,47]$. Furthermore we show in Fig. 3 the $S^{2}$ factors of the ${ }^{8} \mathrm{Be}+\alpha$ components, which are defined below,

$$
S_{[I, l]}^{2}\left(J_{\lambda}^{+}\right)=\int d r\left[r \mathcal{Y}_{[I, l] J}(r)\right]^{2},
$$

where $\mathcal{Y}_{[I, l]_{J}}$ is the reduced width amplitudes of the ${ }^{12} \mathrm{C}\left(J_{\lambda}^{+}\right)$states in the ${ }^{8} \mathrm{Be}(I)+\alpha(l)$ channel. Except for the $0_{4}^{+}$state, all the states have dominant components from the channels $[0, J]_{J}$, which is consistent with ${ }^{8} \mathrm{Be}\left(0^{+}\right)$and $\alpha$ rotation. However, in the $2_{2}^{+}$and $4_{2}^{+}$state, the mixtures of the other components are also found to be large. These suggest that to conclude from there this gives rise to a simple rotational band, is premature [16].

\section{Container evolution for clusters in ${ }^{16} \mathrm{O}$}

The situation in ${ }^{16} \mathrm{O}$ is more complicated than in ${ }^{12} \mathrm{C}$, since even if one $\alpha$ cluster is knocked out, the remaining core configuration is not unique, i.e. many varieties ${ }^{12} \mathrm{C}\left(0_{1}^{+}\right)$, ${ }^{12} \mathrm{C}\left(0_{2}^{+}\right)(3 \alpha)$, etc., are present. Then the special interest is of how these complex cluster states are formed as the increase of excitation energy. The $\alpha+{ }^{12} \mathrm{C}$ cluster structure in 
Table 2. Maxima of the squared overlaps in Eq. (7) for the $0_{I}^{+}-0_{V}^{+}$states, in the four-parameter space $\left(\beta_{1 \perp}, \beta_{1 z}, \beta_{2 \perp}, \beta_{2 z}\right)$. The corresponding parameter values $\left(B_{1 \perp}, B_{1 z}, B_{2 \perp}, B_{2 z}\right)$ are also shown. Those for ${ }^{12} \mathrm{C}$ are also shown, in two-parameter space $\beta_{\perp}$ and $\beta_{z}$, with $\beta_{1}=\beta_{2}$. The corresponding $B_{\perp}$ and $B_{z}$ values are also shown.

\begin{tabular}{cccccccc}
\hline & $O_{\max }$ & $\left(\beta_{1 \perp}, \beta_{1 z}, \beta_{2 \perp} \beta_{2 z}\right)$ & $\left(B_{1 \perp}, B_{1 z}, B_{2 \perp}, B_{2 z}\right)$ & & $O_{\max }$ & $\left(\beta_{1 \perp}, \beta_{1 z}\right)$ & $\left(B_{1 \perp}, B_{1 z}\right)$ \\
\hline $0_{I}^{+}$ & 0.98 & $(1.3,0.1,0.1,2.6 \mathrm{fm})$ & $(2.3,1.4,1.4,3.9 \mathrm{fm})$ & ${ }^{12} \mathrm{C}\left(0_{1}^{+}\right)$ & 0.93 & $(1.9,1.8 \mathrm{fm})$ & $(3.0,2.9 \mathrm{fm})$ \\
$0_{I I}^{+}$ & 0.94 & $(1.8,1.8,3.5,3.6 \mathrm{fm})$ & $(2.9,2.9,5.2,5.3 \mathrm{fm})$ & ${ }^{12} \mathrm{C}\left(2_{1}^{+}\right)$ & 0.90 & $(1.9,0.5 \mathrm{fm})$ & $(3.0,1.6 \mathrm{fm})$ \\
$0_{I I I}^{+}$ & 0.76 & $(2.1,0.7,5.1,0.1 \mathrm{fm})$ & $(3.3,1.7,7.4,1.4 \mathrm{fm})$ & ${ }^{12} \mathrm{C}\left(0_{2}^{+}\right)$ & 0.99 & $(5.6,1.4 \mathrm{fm})$ & $(8.0,2.4 \mathrm{fm})$ \\
$0_{I V}^{I I}$ & 0.84 & $(2.5,1.3,8.3,7.8 \mathrm{fm})$ & $(3.8,2.3,11.8,11.1 \mathrm{fm})$ & & & & \\
$0_{V}^{+}$ & 0.78 & $(5.3,1.9,5.3,1.8 \mathrm{fm})$ & $(7.6,3.0,7.6,2.9 \mathrm{fm})$ & & & & \\
\hline
\end{tabular}

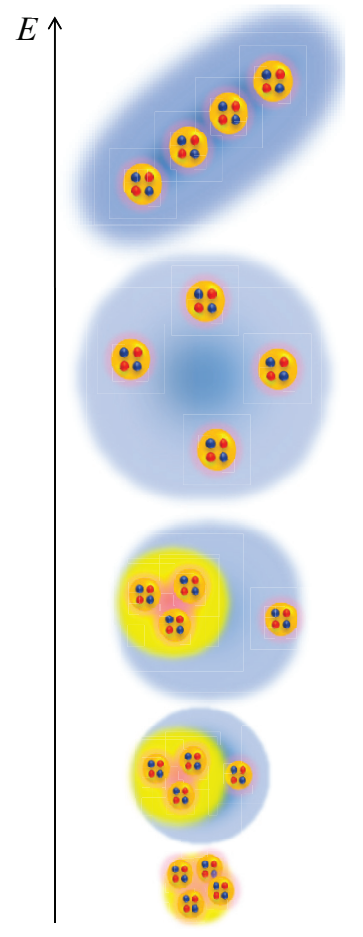

Figure 4. (Color online) "Container" evolution picture for cluster structures in ${ }^{16} \mathrm{O}$.

${ }^{16} \mathrm{O}$ is formed by the activation of cluster degree of freedom in the ground state having a dual property [50,51], i.e. the excitation of relative motion between the $\alpha$ and ${ }^{12} \mathrm{C}$ clusters. The gaslike $4 \alpha$ cluster state is then formed as a result of further excitation of the ${ }^{12} \mathrm{C}$ core to the $3 \alpha$ cluster state, i.e. to the Hoyle state. The path of this cluster evolution is to be shown by the Ikeda diagram. We show in Fig. 4 a schematic picture for the path of cluster evolution along the excitation energy in ${ }^{16} \mathrm{O}$, in terms of the size and shape evolution of containers.

In order to describe the cluster evolution in ${ }^{16} \mathrm{O}$, we employ the following eTHSR wave function, which is a natural extension of what is adopted for the $3 \alpha$ system in the previous section,

$$
\begin{aligned}
& \Phi_{4 \alpha}^{J=0}\left(\boldsymbol{\beta}_{1}, \boldsymbol{\beta}_{2}\right) \\
& =\hat{P}^{J=0} \mathcal{A}\left[\exp \left\{-2 \sum_{k}^{x, y, z}\left(\frac{\mu_{1} \xi_{1 k}^{2}+\mu_{2} \xi_{2 k}^{2}}{B_{1 k}^{2}}-\frac{\mu_{3} \xi_{3 k}^{2}}{B_{2 k}^{2}}\right)\right\} \prod_{i=1}^{4} \phi\left(\alpha_{i}\right)\right],
\end{aligned}
$$

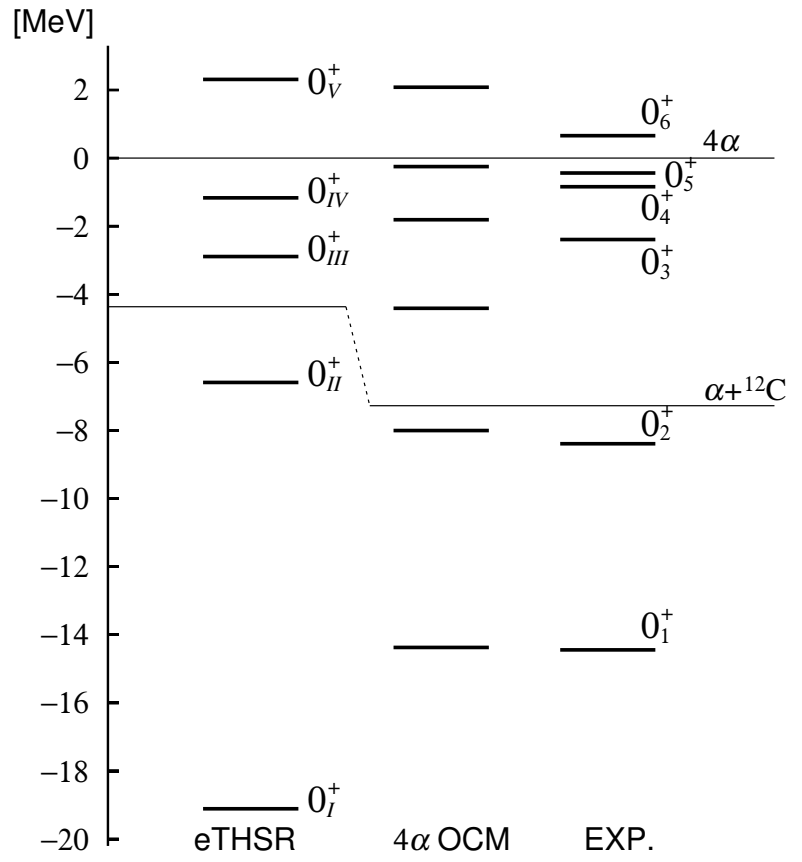

Figure 5. Energy spectra of the low-lying $J^{\pi}=0^{+}$states calculated with the extended THSR ansatz. The corresponding observed spectrum (Exp.) [48, 49] and result by the $4 \alpha$ OCM [11] are also shown.

where $\xi_{i}$ is again the Jacobi coordinates between the $\alpha$ particles, and $\mu_{i}=i /(i+1)$, for $i=1,2,3$. While the parameter $b$ characterizes the size of the constituent $\alpha$ particle, the parameters $\boldsymbol{\beta}_{1}$ and $\boldsymbol{\beta}_{2}$ characterize the size and shape of a container, in which the $\alpha$ clusters are confined. We also assume the axial symmetry $\beta_{i \perp} \equiv \beta_{i x}=\beta_{i y}$, so as to deal with the four parameters, $\beta_{1 \perp}, \beta_{1 z}, \beta_{2 \perp}, \beta_{2 z}$, in the practical calculations.

In Fig. 5, the calculated energy spectrum for $J^{\pi}=0^{+}$ states is shown. The corresponding experimental data and result by the $4 \alpha$ Orthogonality Condition Model (OCM) calculation [11] are also shown. The solution of HillWheeler equation with the radius constraint method is shown.

The $4 \alpha$ OCM calculation gives six $0^{+}$states. The $0_{6}^{+}$ state has the $4 \alpha$ condensate character and the $0_{2}^{+}-0_{5}^{+}$states all have $\alpha+{ }^{12} \mathrm{C}$ cluster structures. i.e. $\alpha(S)+{ }^{12} \mathrm{C}\left(0_{1}^{+}\right)$, $\alpha(D)+{ }^{12} \mathrm{C}\left(2_{1}^{+}\right), \alpha(S)+{ }^{12} \mathrm{C}\left(0_{1}^{+}\right)$, and $\alpha(P)+{ }^{12} \mathrm{C}\left(1^{-}\right)$cluster structures, respectively. Since in the present eTHSR wave function of Eq. (6) the $\alpha$ clusters occupy positive parity or- 
bits, such a state as having the $\alpha(P)+{ }^{12} \mathrm{C}\left(1^{-}\right)$cluster structure, like the $0_{5}^{+}$state in the OCM calculation, is missing.

We calculate the r.m.s. radii for the five states, whose values are $2.7,3.2,3.3,4.9$, and $4.9 \mathrm{fm}$, respectively from the ground state to the $0_{V}^{+}$state, and namely as the states are excited, the r.m.s. radius becomes larger. This indicates that the higher the excitation energy is, the more evolved the clustering is. The evolution of the clustering can be described by solving the Hill-Wheeler equation concerning the model parameters $\boldsymbol{\beta}_{1}$ and $\boldsymbol{\beta}_{2}$.

This respect is made much clearer by calculating the following squared overlap:

$$
\mathcal{O}_{\lambda}\left(\boldsymbol{\beta}_{1}, \boldsymbol{\beta}_{2}\right)=\left|\left\langle\widetilde{\Phi}_{\lambda}^{J=0}\left(\boldsymbol{\beta}_{1}, \boldsymbol{\beta}_{2}\right) \mid \Psi_{\lambda}\right\rangle\right|^{2},
$$

with $\Psi_{\lambda}$ the $\lambda$ th eigenfunction and $\widetilde{\Phi}_{\lambda}^{J=0}\left(\boldsymbol{\beta}_{1}, \boldsymbol{\beta}_{2}\right)$ normalized single eTHSR wave function in a space orthogonal to the lower eigenstates, i.e.

$$
\widetilde{\Phi}_{\lambda}^{J=0}\left(\boldsymbol{\beta}_{1}, \boldsymbol{\beta}_{2}\right)=\mathcal{N}_{\lambda} \hat{P}_{\lambda} \Phi_{4 \alpha}^{J=0}\left(\boldsymbol{\beta}_{1}, \boldsymbol{\beta}_{2}\right)
$$

where $\hat{P}_{\lambda}=1-\sum_{i=1}^{\lambda-1}\left|\Psi_{i}\right\rangle\left\langle\Psi_{i}\right|$ with $\lambda=I, \cdots, I V$, and $\hat{P}_{V}=1-\sum_{i=1}^{6}\left|\Psi_{i}\right\rangle\left\langle\Psi_{i}\right|$, and $\mathcal{N}_{\lambda}$ are the corresponding normalization constants. This quantity shows how these states $\Psi_{\lambda}(\lambda=I, \cdots, V)$ are represented by single eTHSR configurations, and therefore, gives direct information of whether the container structure is realized and what kind of containers are realized.

In Table 2 , the maximal values and $\boldsymbol{\beta}_{1}$ and $\boldsymbol{\beta}_{2}$ parameter values to give the maxima in the squared overlap are listed. The corresponding $\boldsymbol{B}_{1}$ and $\boldsymbol{B}_{2}$ values are also shown. It is now well known that the single $3 \alpha$ THSR wave function can very precisely describe the ground state and excited states of ${ }^{12} \mathrm{C}$. Then we also show the maximum values of the squared overlap of the $0_{1}^{+}, 2_{1}^{+}$and $0_{2}^{+}$ states in ${ }^{12} \mathrm{C}$ with the single $3 \alpha$ THSR configuration in $\boldsymbol{\beta}_{1}=\boldsymbol{\beta}_{2}$ parameter space.

In the ground state, $3 \alpha$ clusters are in an oblately deformed and very compact container with $\beta_{1 \perp} \gg \beta_{1 z}$, while the remaining $\alpha$ cluster is in a prolately deformed and very compact container with $\beta_{2 \perp} \ll \beta_{2 z}$. This means that the first $3 \alpha$ clusters move in a $x y$-plane and the last one moves in $z$-direction. This supports the idea that the ground state has a tetrahedral shape of the $4 \alpha$ clusters proposed by several authors $[52,53]$. Our calculation indicates that this configuration is contained in the $0_{I}^{+}$state by $98 \%$.

In the $0_{I I}^{+}$state, the $3 \alpha$ clusters are in a spherical container with $\beta_{1 \perp} \sim \beta_{1 z}$. The fourth $\alpha$ cluster is put into a larger size container with spherical shape, i.e. $\beta_{2 \perp} \sim$ $\beta_{2 z}>\beta_{1 \perp} \sim \beta_{1 z}$. In particular, the parameter set $\left(\beta_{1 \perp}, \beta_{1 z}\right)=(1.8,1.8 \mathrm{fm})$ is almost the same as that for ${ }^{12} \mathrm{C}$, i.e. $\left(\beta_{\perp}, \beta_{z}\right)=(1,9,1.8 \mathrm{fm})$. This means that the first $3 \alpha$ clusters are confined in a compact container to form the ground state of ${ }^{12} \mathrm{C}$, since the ${ }^{12} \mathrm{C}\left(0_{1}^{+}\right)$state can be very precisely described by the single configuration with these parameter values. The fourth $\alpha$ cluster moves in a larger spherical container, because of $\left(\beta_{2 \perp}, \beta_{2 z}\right)=(3.5,3.6 \mathrm{fm})$, which gives the largest squared overlap $94 \%$. This is the new interpretation of the $\alpha+{ }^{12} \mathrm{C}$ cluster structure, which is consistent with the traditional understanding that the $\alpha$ cluster orbits in an $S$-wave around the ${ }^{12} \mathrm{C}\left(0_{1}^{+}\right)$core.
The $0_{I I I}^{+}$state is similar to the $0_{I I}^{+}$state but both containers are not spherical but deformed. The $\boldsymbol{\beta}_{1}$ parameter takes almost the same value as that of the isolated ${ }^{12} \mathrm{C}\left(2^{+}\right)$state, which means that the first $3 \alpha$ clusters form the ${ }^{12} \mathrm{C}\left(2^{+}\right)$state, since the state is described by the single parameter value of $\boldsymbol{\beta}$. The configuration of the remaining $\alpha$ cluster $\left(\beta_{2 \perp}, \beta_{2 z}\right)=(5.1,0.1 \mathrm{fm})$, giving the largest value $76 \%$, means that the $\alpha$ cluster moves in a deformed and larger container. This is present understanding of the $\mathrm{O}_{3}^{+}$state, which is conventionally considered to have the $\alpha(D)+{ }^{12} \mathrm{C}\left(2^{+}\right)$structure.

In the $0_{I V}^{+}$state one can see that the $3 \alpha$ clusters are put in slightly larger container than that for the ${ }^{12} \mathrm{C}\left(0_{1}^{+}\right)$state, which is slightly deformed in a oblate shape. The fourth $\alpha$ cluster, however, moves in a much larger and almost spherical container, like a satellite. This configuration expresses the $0_{I V}^{+}$state dominantly by $84 \%$. This means that the second container characterized by $\boldsymbol{\beta}_{2}$ is further evolved from that in the $0_{I I}^{+}$state. We can say that this state corresponds to the $0_{4}^{+}$state in the former $4 \alpha$ OCM calculation, which predicts the $\alpha+{ }^{12} \mathrm{C}\left(0_{1}^{+}\right)$higher nodal structure for the state.

The $0_{V}^{+}$state is the most interesting. All the $\alpha$ clusters occupy an identical orbit, with $\left(\beta_{1 \perp}, \beta_{1 z}, \beta_{2 \perp}, \beta_{2 z}\right)=$ $(5.3,1.9,5.3,1.8 \mathrm{fm})$. This is qualified to call the $\alpha$ condensation. This configuration is contained in this state by $78 \%$, which is still very large. Furthermore, this container is very close to the one of the Hoyle state, with $\left(\beta_{1 \perp}, \beta_{1 z}\right)=(5.6,1.4 \mathrm{fm})$. This means that the $0_{V}^{+}$state is regarded as the Hoyle analog state, in which the fourth $\alpha$ cluster is also put into the container occupied with the $3 \alpha$ clusters in the Hoyle state. The large size of this container indicates that the $4 \alpha$ clusters are loosely coupled with each other and configured like a gas. Note that the $4 \alpha$ condensate state is also predicted by the $4 \alpha$ OCM calculation slightly above the $4 \alpha$ threshold, as the $0_{6}^{+}$state.

These results tell us that the evolution of cluster structures is described by the container evolution with respect to its size and shape. The reason why the container evolution arises is the orthogonality to the lower states, which is explicitly taken into account in the definition of the single configuration $\widetilde{\Phi}_{k}^{J=0}$ in Eq. (7). The orthogonality condition prevents a higher state configuration from overlapping with the lower-states more compact configurations. It thus plays a role as a repulsive core and is considered to give the container evolution.

\section{Conclusion}

We proposed a new concept, "container" picture, to describe general cluster structures. This idea comes with the so-called THSR ansatz, which was originally introduced to explain the gaslike cluster states like the $\alpha$ condensates. We further proposed a "container evolution" picture, in which the cluster evolution is caused by the container evolution. We demonstrated this idea in ${ }^{16} \mathrm{O}$ nucleus and showed that the cluster formation, from the ground and $\alpha+{ }^{12} \mathrm{C}$ cluster states to the $4 \alpha$ gaslike state, is nicely reproduced by the eTHSR wave function. This idea is promising for heavier nuclei and is to deepen the understanding of cluster formation described by the Ikeda diagram. 


\section{References}

[1] K. Ikeda, H. Horiuchi, S. Saito, Suppl. Prog. Theor. Phys. 68, 1 (1980)

[2] T.K. Rana et al., Phys. Rev. C 88, 021601(R) (2013)

[3] M. Freer, H.O.U. Fynbo, Prog. Part. Nucl. Phys. 78, 1 (2014)

[4] M. Itoh et al., Phys. Rev. Lett. 113, 102501 (2014)

[5] A. Tohsaki, H. Horiuchi, P. Schuck, G. Röpke, Phys. Rev. Lett. 87, 192501 (2001)

[6] Y. Funaki, H. Horiuchi, A. Tohsaki, P. Schuck, G. Röpke, , Prog. Theor. Phys. 108, 297 (2002)

[7] Y. Funaki, A. Tohsaki, H. Horiuchi, P. Schuck, G. Röpke, Phys. Rev. C 67, 051306(R) (2003)

[8] T. Yamada, P. Schuck, Phys. Rev. C 69, 024309 (2004)

[9] T. Yamada, P. Schuck, Eur. Phys. J. A 26, 185 (2005)

[10] Y. Funaki, A. Tohsaki, H. Horiuchi, P. Schuck, G. Röpke, Eur. Phys. J. A 28, 259 (2006)

[11] Y. Funaki, T. Yamada, H. Horiuchi, G. Röpke, P. Schuck, A. Tohsaki, Phys. Rev. Lett. 101, 082502 (2008)

[12] Y. Funaki, H. Horiuchi, W. von Oertzen, G. Röpke, P. Schuck, A. Tohsaki, T. Yamada, Phys. Rev. C 80, 064326 (2009)

[13] B. Zhou, Y. Funaki, H. Horiuchi, Z.Z. Ren, G. Röpke, P. Schuck, A. Tohsaki, C. Xu, T. Yamada, Phys. Rev. Lett. 110, 262501 (2013)

[14] T. Suhara, Y. Funaki, B. Zhou, H. Horiuchi, A. Tohsaki, Phys. Rev. Lett. 112, 062501 (2014)

[15] Y. Funaki, H. Horiuchi, A. Tohsaki, Prog. Part. Nucl. Phys. 82, 78 (2015)

[16] Y. Funaki, Phys. Rev. C 92, 021302(R) (2015)

[17] Y. Funaki, Phys. Rev. C 94, 024344 (2016)

[18] Y. Funaki, Phys. Rev. C 97, 021304(R) (2018)

[19] H. Horiuchi, K. Ikeda, Prog. Theor. Phys. 40, 277 (1968)

[20] B. Zhou, Y. Funaki, H. Horiuchi, Z.Z. Ren, G. Röpke, P. Schuck, A. Tohsaki, C. Xu, T. Yamada, Phys. Rev. C 89, 034319 (2014)

[21] Y. Funaki, T. Yamada, E. Hiyama, B. Zhou, K. Ikeda, Prog. Theor. Exp. Phys. 2014, 113D01 (2014)

[22] M. Lyu, Z.Z. Ren, B. Zhou, Y. Funaki, H. Horiuchi, G. Röpke, P. Schuck, A. Tohsaki, C. Xu, T. Yamada, Phys. Rev. C 91, 014313 (2015)

[23] M. Lyu, Z.Z. Ren, B. Zhou, Y. Funaki, H. Horiuchi, G. Röpke, P. Schuck, A. Tohsaki, C. Xu, T. Yamada, Phys. Rev. C 93, 054308 (2016)

[24] Y. Funaki, M. Isaka, E. Hiyama, T. Yamada, K. Ikeda, Phys. Lett. B 773, 336 (2017)

[25] Q. Zhao, Z.Z. Ren, M. Lyu, H. Horiuchi, Y. Funaki, G. Röpke, P. Schuck, A. Tohsaki, C. Xu, T. Yamada et al., Phys. Rev. C 97, 054323 (2018)

[26] Y. Funaki, A. Tohsaki, H. Horiuchi, P. Schuck, G. Röpke, Eur. Phys. J. A 24, 321 (2005)

[27] Y. Funaki, H. Horiuchi, A. Tohsaki, Prog. Theor. Phys. 115, 115 (2006)

[28] Y. Funaki, T. Yamada, A. Tohsaki, H. Horiuchi, G. Röpke, P. Schuck, Phys. Rev. C 82, 024312 (2010)

[29] E. Uegaki, S. Okabe, Y. Abe, H. Tanaka, Prog. Theor. Phys. 57, 1262 (1977)

[30] P. Descouvemont, D. Baye, Phys. Rev. C 36, 54 (1987)

[31] M. Kamimura, Nucl. Phys. A 351, 456 (1981)

[32] M. Itoh et al., Nucl. Phys. A 738, 268 (2004)

[33] M. Freer et al., Phys. Rev. C 80, 041303(R) (2009)

[34] M. Itoh et al., Phys. Rev. C 84, 054308 (2011)

[35] H.O.U. Fynbo, M. Freer, Physics 4, 94 (2011)

[36] W.R. Zimmerman, N.E. Destefano, M. Freer, M. Gai, F.D. Smit, Phys. Rev. C 84, 027304 (2011)

[37] W.R. Zimmerman et al., Phys. Rev. Lett. 110, 152502 (2013)

[38] M. Freer et al., Phys. Rev. C 83, 034314 (2011)

[39] M. Itoh et al., J. Phys. Conf. Ser. 436, 012006 (2013)

[40] C. Kurokawa, K. Kato, Phys. Rev. C 71, 021301 (2005)

[41] B. Zhou, A. Tohsaki, H. Horiuchi, Z.Z. Ren, Phys. Rev. C 94, 044319 (2016)

[42] Y. Kanada-En'yo, Prog. Theor. Phys. 117, 655 (2007)

[43] M. Chernykh, H. Feldmeier, T. Neff, P. von Neumann-Cosel, A. Richter, Phys. Rev. Lett. 98, 032501 (2007)

[44] R. Bijker, F. Iachello, Phys. Rev. C 61, 067305 (2000)

[45] D.J. Mariń-Laḿbarri, R. Bijker, M. Freer, M. Gai, T. Kokalova, D. Parker, C. Wheldon, Phys. Rev. Lett. 113, 012502 (2014)

[46] S. Ohkubo, Y. Hirabayashi, Phys. Lett. B 684, 127 (2010)

[47] Y. Funaki, T. Yamada, H. Horiuchi, G. Röpke, P. Schuck, A. Tohsaki, Prog. Theor. Phys. Suppl. 196, 439 (2012)

[48] F. Ajzenberg-Selove, Nucl. Phys. A 460, 1 (1986)

[49] T. Wakasa et al., Phys. Lett. B 653, 173 (2007)

[50] T. Yamada, Y. Funaki, H. Horiuchi, K. Ikeda, A. Tohsaki, Prog. Theor. Phys. 120, 1139 (2008)

[51] T. Yamada, Y. Funaki, T. Myo, H. Horiuchi, K. Ikeda, G. Röpke, P. Schuck, A. Tohsaki, Phys. Rev. C 85, 034315 (2012)

[52] R. Bijker, F. Iachello, Phys. Rev. Lett. 112, 152501 (2014)

[53] Y. Kanada-En'yo, Phys. Rev. C 96, 034306 (2017) 